\title{
Assessment of Medical Records Documentation in Secondary Health Care Facilities in Kafr El-Sheikh and El-Mahalla El-Kubra Cities
}

\author{
HEBAT ALLAH A. AHMED, M.Sc.; RANYA M. EL-SALAMI, M.D.; KHALIL M. AYAD, M.D. and \\ ABD EL-AZIZ F. EL-DEEB, M.D. \\ The Department of Public Health \& Community Medicine, Faculty of Medicine, Tanta University
}

\begin{abstract}
Background: Accurate, timely and accessible health care data play an important role in the planning, development and maintenance of health care services.

Aim of Study: This study was undertaken to assess the quality of medical records documentation in three healthcare facilities, two of them in Kafr El-Sheikh City (Kafr El-Sheikh General Hospital and El Obor Health Insurance Hospital) and the third one in El-Mahalla El-Kubra City (El-Mahalla ElKubra General Hospital, Gharbia Governerate).

Subjects and Methods: The medical record sample calculated was (200) record sample from El-Mahalla El-Kubra General Hospital, (300) record sample from Kafr El-Sheikh General Hospital and (350) record sample from El Obor Health Insurance Hospital. The calculated record sample for each hospital was selected from the discharge logs of "2017" using the systematic random sampling method.

Results: General consent form and discharge summary sheet were completely absent from the medical records of ElObor Health Insurance Hospital. Almost all documentation assessment items of patient identification part were present in more than two third of checked records in El-Obor Health Insurance Hospital. The documentation assessment items of front sheet and discharge summary sheet were not present in more than the half of reviewed records at Kafr El-Sheikh General Hospital. Recording of clinical progress notes daily was not present in more than two third of reviewed records in the three hospitals. Recording of nursing progress notes within each shift was present in the majority of reviewed records $(80 \%)$ in the three studied hospitals.
\end{abstract}

Conclusion: There was considerable variations between the three studied hospitals according to documentation quality.

Key Words: Medical records - Documentation - Secondary health care facilities.

\section{Introduction}

MEDICAL record is an orderly and chronologically written document which stores data concerning the patient and his care during hospitalization.

Correspondence to: Dr. Hebat Allah A. Ahmed,

The Department Public Health \& Community Medicine, Faculty of Medicine, Tanta University
The complete medical record must contain sufficient data to clearly identify the patient, support the diagnosis, justify treatment received and accurately document the outcome $[1,2]$.

The World Health Organization (WHO) stated that the proper collection, management and use of information within healthcare systems will determine the system's effectiveness in detecting health problems, defining priorities, identifying innovative solutions and allocating resources to improve health outcomes [3].

There are many problems with the documentation systems worldwide, like missing the data, incorrect data, duplication of patient's record and unreadable entries. Such an unreliable and poor quality data should not be used in making decisions in healthcare management. Moreover, the lack of correct and timely entry data can lead to poor choices in clinical practice, medication errors, inappropriate repeating of tests, unnecessary referrals, and the waste of time and other resources [4-5].

\section{The rationale of the study:}

This study is designed as a situation analysis of the quality of documentation of medical records in some Egyptian health care facilities, to help appropriate decisions making and institution of proper guidelines \& interventions aiming at quality improvement of patient record.

\section{Subjects and Methods}

Study design: It is a cross-sectional study.

Study site and period: The study was conducted in the Medical Records Department of three health care facilities, two of them in Kafr El-Sheikh City (Kafr El-Sheikh General Hospital and El-Obor Health Insurance Hospital) and the third one in ElMahalla El-Kubra City (El-Mahalla El-Kubra Gen- 
eral Hospital, Gharbia Governerate) between March 2017 and completed by October 2018.

Sample size and technique: The medical record sample calculated was two hundred record sample from El-Mahalla El-Kubra General Hospital, three hundred record sample from Kafr El-Sheikh General Hospital and three hundred and fifteen record sample from El-Obor Health Insurance Hospital. This sample represents approximately $(1 \%)$ of the discharges in each hospital for "2017". The calculated record sample for each hospital was selected from the discharge logs of "2017" using the systematic random sampling method. For each case, the patient name and the medical record number were listed and the sample list given to the head of Medical Record Department to facilitate retrieval of the chosen records. If an assigned record was missing, it was substituted by the case next to it in the discharge log.

Study tools and data collection technique: Data were collected through revising of properly selected random sample of paper-based medical records of discharged patients during the year 2017 to fill assessment checklist tool. The assessment checklist sheet containing data regarding Quantitative assessment and Qualitative assessment of medical records:

1-Quantitative assessment: The medical record was considered quantitatively complete if it include all basic forms (essential forms that have to be included and completed for each patient admitted to hospital during period of hospitalization). The following ten basic forms were checked for every record included in the study; (Admission and discharge form (front sheet), Consent form, Discharge summary sheet, Medical history sheet, Physical examination sheet, Clinical progress notes sheet. Treatment orders sheet, Nursing notes sheet, Graphic sheet (temp, pulse) and Routine diagnostic reports).

2- Qualitative assessment: Reviewing of contents of basic medical record forms using a criteria checklist for each form. Recorded items were checked as positive findings while missing or illegible ones were considered as negative findings. The following are details of criteria items for each form:

- Patient identification part: Includes patient 's full name, patient 's full address, unique patient identifiers, gender, age, occupation and marital status.

- Admission and discharge form (front sheet): includes provisional diagnosis, time and date of admission, final diagnosis and time and date of discharge.
- General consent form: Includes signing of consent form by the patient or his relatives.

- Discharge summary sheet: Includes patient status at discharge, final diagnosis, operative procedures and intervention, discharge Medications, discharge instructions, follow-up appointments.

- Medical history and physical examination sheet: Includes patient chief complaint, history of present illness, previous hospital admissions and surgery, past history, family history, personal habits and complete physical assessment of all systems of the body.

- Clinical progress notes sheet: Includes recording of progress notes at least once daily.

- Treatment orders sheet: Includes legible medication name, dose, route and frequency.

- Nursing notes sheet: Includes recording of nursing notes findings each shift and items for Medication administration sheet (legible medication name, dose, route and frequency).

- Graphic sheet: Includes flow sheet documentation assessment (blood pressure, heart rate, temperature and respiration).

\section{Ethical consideration:}

- Ethical considerations of the study were carried out according to that of Tanta Faculty of Medicine. The study was approved by Ethical Committee of the Faculty of Medicine; Tanta University. An official permission letter was obtained and directed to administrators of selected hospitals.

- Formal approval from managers of hospitals was taken to work in the medical record department.

- Medical record personnel were oriented about the purpose and procedure of the study.

- The researcher was the only person with the full authority to access and audit the patients' records.

\section{Statistical analysis:}

Sorting and analysis of data were performed by using Statistical Package for Social Sciences (SPSS) version 21. The qualitative data were prescribed using number and percent, Chi-square test was used for analysis and when it was found inappropriate, it was replaced by Monte Carlo exact test or Fisher's exact test.

\section{Results}

Comparison of existence of the basic forms in the checked medical records in the three studied hospitals: Front sheet, medical history sheet, physical examination sheet, clinical progress notes 
sheet, treatment order sheet, nursing notes sheet and graphic sheet were completely present in all records $(100 \%)$ of the studied hospitals. Consent form and discharge summary sheet were completely absent in all checked records (100\%) of El-Obor Health Insurance Hospital. Routine diagnostic reports were present in the majority of the checked records $(60 \%)$ in the three studied hospitals.

Comparison of completeness of documentation for items of patient identification part in the three studied hospitals (Table 1): There was a statistical significant difference of documentation of all items of patient identification form between the three hospitals. Patient's full name was present in all checked records (100\%) of El-Obor Health Insurance Hospital. Patient's full address was present in $64.5 \%$ records of El-Mahalla El-Kubra General Hospital. Unique patient identifiers were present in $8.5 \%$ records of Kafr El-Sheikh General Hospital. Documentation of gender, occupation and marital status of the admitted patients were not present completely in all reviewed records of ElMahalla El-Kubra General Hospital.

Comparison of completeness of documentation for items of front sheet form in the three studied hospitals (Table 2): There was a statistical significant difference of documentation of all items of front sheet form between the three hospitals. Patient's location and signing of front sheet were present completely in all checked records of ElMahalla El-Kubra General Hospital. Documentation of provisional diagnosis and final diagnosis were present in most of El-Mahalla El-Kubra General Hospital checked records (96\%) and (78\%) respectively. Documentation of time of admission and time of discharge were least frequent in Kafr El-Sheikh General Hospital, they were not present in most of records (83.5\%) and (100\%) respectively.

Comparison of completeness of documentation for items of discharge summary in the three studied hospitals (Table 3): Documentation of patient status at discharge and final diagnosis were present in majority of records $(81 \%)$ and $(75 \%)$ respectively in El-Mahalla El-Kubra General Hospital. Discharge instructions to the patient were not present in all reviewed records of Mahalla El-Kubra General Hospital and in most of records (98.5\%) at Kafr El-Sheikh General Hospital. Signature of documented physician in discharge summary was present in $97 \%$ of checked records in Mahalla ElKubra General Hospital.

Comparison of completeness of documentation for items of general consent forms in the three studied hospitals: Signing of general consent form was fulfilled in all records of El-Mahalla El-Kubra General Hospital and in 63\% records of Kafr ElSheikh General Hospital. There was a statistical significant difference of signing of general consent form at ( $p$-value $=.0000)$.

Comparison of completeness of documentation for items of medical history sheet and physical examination form in the three studied hospitals (Table 4): There was a statistical significant difference of documentation of all items of medical history and physical examination sheet between the three hospitals. Rate of documentation of patient chief complaint was the highest in Kafr El-Sheikh General Hospital in $92.5 \%$ of records. History of present illness was not documented in all checked records of El-Mahalla El-Kubra General Hospital. Rate of documentation of past history was the lowest in El-Mahalla El-Kubra General Hospital, it was not present in $72 \%$ records. Personal habits was not documented in $95.5 \%$ of records in ElObor Health Insurance Hospital. Previous hospital admissions and surgery was not documented in majority of records (70-100\%) in the three studied hospitals.

Comparison of completeness of documentation for items of treatment orders sheet in the three studied hospitals: Legible medication name was present in more than $90 \%$ of checked records of the three studied hospitals. Documentation of frequency of administration was fulfilled in around $90 \%$ of records in the three studied hospitals. Documentation of medications dose was fulfilled in majority of checked records (70-80\%) in the three studied hospitals. Physician's signature was present in more than $95 \%$ of checked records in the three studied hospitals. There was a statistical significant difference of documentation of all items of treatment order sheet except (frequency of administered medications) between the three hospitals.

Comparison of completeness of documentation for items of clinical progress notes sheet in the three studied hospitals: Documentation of clinical progress notes daily was not fulfilled in $91 \%$ records of El-Mahalla El-Kubra General Hospital, in $81.5 \%$ records of El-Obor Health Insurance Hospital and in $69 \%$ records of Kafr El-Sheikh General Hospital.

Comparison of completeness of documentation for items of nursing notes sheet in the three studied hospitals (Table 5): The rate of recorded nursing notes each shift was highest in El-Mahalla ElKubra General Hospital, in $88 \%$ of records. There was a statistical significant difference of documen- 
tation of all items of medication administered by nurses between the three hospitals.

Comparison of completeness of documentation for items of flow sheet in the three studied hospitals (Fig. 1): Documentation of four elements of vital signs were highest in El-Mahalla El-Kubra General
Hospital, they were present in majority of records (70-90\%). Documentation of blood pressure was not present in $97 \%$ records of El-Obor Health Insurance Hospital. There was a statistical significant difference of documentation of different elements of vital signs, except heart rate between the three hospitals.

Table (1): Comparison of completeness of documentation for items of patient identification part in the three studied hospitals.

\begin{tabular}{|c|c|c|c|c|c|}
\hline Variable & $\begin{array}{c}\text { Kafr } \\
\text { El-Sheikh } \\
\text { General Hospital }\end{array}$ & $\begin{array}{c}\text { El-Obor } \\
\text { Health Insurance } \\
\text { Hospital }\end{array}$ & $\begin{array}{c}\text { El-Mahalla } \\
\text { El-Qubra } \\
\text { General Hospital }\end{array}$ & $X^{2}$ & $p$ \\
\hline \multicolumn{6}{|c|}{ Patient 's full name: } \\
\hline No $(\%)$ & $290(96.5)$ & $350(100)$ & $184(92)$ & & \\
\hline $\begin{array}{l}\text { Not present } \\
\text { No }(\%)\end{array}$ & $10(3.5)$ & $0(0)$ & $16(8)$ & 5.3 & $0.021 *$ \\
\hline $\begin{array}{l}\text { Total } \\
\text { No }(\%)\end{array}$ & $300(100)$ & $350(100)$ & $200(100)$ & & \\
\hline $\begin{array}{l}\text { Patient 'sfull ac } \\
\text { Present } \\
\text { No }(\%)\end{array}$ & $74(24.5)$ & $52(15)$ & $129(64.5)$ & & \\
\hline $\begin{array}{l}\text { Not present } \\
\text { No }(\%)\end{array}$ & $226(75.5)$ & $298(85)$ & $71(35.5)$ & MCET** $^{* *}$ & $.0000^{*}$ \\
\hline $\begin{array}{l}\text { Total } \\
\text { No }(\%)\end{array}$ & $300(100)$ & $350(100)$ & $200(100)$ & & \\
\hline \multicolumn{6}{|c|}{ Unique patient identifier: } \\
\hline No $(\%)$ & $25(8.5)$ & $332(95)$ & $200(100)$ & & \\
\hline Not present & & & & & \\
\hline $\begin{array}{l}\text { No }(\%) \\
\text { Total }\end{array}$ & $275(91.5)$ & $18(5)$ & $0(0)$ & MCET** & $.0000 *$ \\
\hline No $(\%)$ & $300(100)$ & $350(100)$ & $200(100)$ & & \\
\hline \multicolumn{6}{|l|}{ Gender: } \\
\hline No $(\%)$ & $209(69.5)$ & $280(80)$ & $0(0)$ & & \\
\hline $\begin{array}{l}\text { Not present } \\
\text { No }(\%)\end{array}$ & $91(30.5)$ & $70(20)$ & $200(100)$ & MCET $* *^{*}$ & $.0000^{*}$ \\
\hline $\begin{array}{l}\text { Total } \\
\text { No }(\%)\end{array}$ & $300(100)$ & $350(100)$ & $200(100)$ & & \\
\hline \multicolumn{6}{|l|}{ Age: } \\
\hline $\begin{array}{l}\text { Present } \\
\text { No }(\%)\end{array}$ & $251(83.5)$ & $343(98)$ & $200(100)$ & & \\
\hline $\begin{array}{l}\text { Not present } \\
\text { No }(\%)\end{array}$ & $49(16.5)$ & $7(2)$ & $0(0)$ & 38.9 & $.0000^{*}$ \\
\hline $\begin{array}{l}\text { Total } \\
\text { No }(\%)\end{array}$ & $300(100)$ & $350(100)$ & $200(100)$ & & \\
\hline $\begin{array}{l}\text { Occupation: } \\
\text { Present }\end{array}$ & & & & & \\
\hline $\begin{array}{c}\text { No }(\%) \\
\text { Not present }\end{array}$ & $80(26.5)$ & $298(85)$ & $0(0)$ & & \\
\hline $\begin{array}{l}\text { No }(\%) \\
\text { Total }\end{array}$ & $220(73.5)$ & $52(15)$ & $200(100)$ & MCET** $^{* *}$ & $.0000 *$ \\
\hline No $(\%)$ & $300(100)$ & $350(100)$ & $200(100)$ & & \\
\hline $\begin{array}{l}\text { Marital status: } \\
\text { Present }\end{array}$ & & & & & \\
\hline $\begin{array}{c}\text { No }(\%) \\
\text { Not present }\end{array}$ & $174(58)$ & $252(72)$ & $0(0)$ & & \\
\hline No $(\%)$ & $126(42)$ & $98(28)$ & $200(100)$ & MCET** & $.0000^{*}$ \\
\hline $\begin{array}{l}\text { Total } \\
\text { No }(\%)\end{array}$ & $300(100)$ & $350(100)$ & $200(100)$ & & \\
\hline
\end{tabular}


Table (2): Comparison of completeness of documentation for items of front sheet form in the three studied hospitals

\begin{tabular}{|c|c|c|c|c|c|}
\hline Variable & $\begin{array}{c}\text { Kafr } \\
\text { El-Sheikh } \\
\text { General Hospital }\end{array}$ & $\begin{array}{c}\text { El-Obor } \\
\text { Health Insurance } \\
\text { Hospital }\end{array}$ & $\begin{array}{c}\text { El-Mahalla } \\
\text { El-Qubra } \\
\text { General Hospital }\end{array}$ & $\mathrm{X}^{2}$ & $p$ \\
\hline \multicolumn{6}{|c|}{ Patient's location: } \\
\hline No $(\%)$ & $131(43.5)$ & 346 (99) & $200(100)$ & \multirow{3}{*}{ MCET** } & \multirow{3}{*}{$0.000^{*}$} \\
\hline $\begin{array}{l}\text { Not present } \\
\text { No }(\%)\end{array}$ & $169(56.5)$ & $4(1)$ & $0(0)$ & & \\
\hline $\begin{array}{l}\text { Total } \\
\text { No }(\%)\end{array}$ & $300(100)$ & $350(100)$ & $200(100)$ & & \\
\hline \multicolumn{6}{|c|}{ Provisional diagnosis: } \\
\hline $\begin{array}{c}\text { No }(\%) \\
\text { Not presen }\end{array}$ & $147(49)$ & $322(92)$ & $192(96)$ & \multirow{3}{*}{ MCET** $^{* *}$} & \multirow{3}{*}{$.0000^{*}$} \\
\hline $\begin{array}{l}\text { No }(\%) \\
\text { Total }\end{array}$ & $153(51)$ & $28(8)$ & $8(4)$ & & \\
\hline No $(\%)$ & $300(100)$ & $350(100)$ & $200(100)$ & & \\
\hline \multicolumn{6}{|c|}{$\begin{array}{l}\text { Time of admission: } \\
\text { Present }\end{array}$} \\
\hline No $(\%)$ & $50(16.5)$ & $296(84.5)$ & $167(83.5)$ & \multirow{3}{*}{$\mathrm{MCET}^{* *}$} & \multirow{3}{*}{$.0000^{*}$} \\
\hline $\begin{array}{l}\text { Not present } \\
\text { No }(\%)\end{array}$ & $250(83.5)$ & $54(15.5)$ & $33(16.5)$ & & \\
\hline $\begin{array}{l}\text { Total } \\
\text { No }(\%)\end{array}$ & $300(100)$ & $350(100)$ & $200(100)$ & & \\
\hline \multicolumn{6}{|c|}{ Date of admission: } \\
\hline $\begin{array}{c}\text { No }(\%) \\
\text { Not presen }\end{array}$ & $255(85)$ & $326(93)$ & $192(96)$ & \multirow{2}{*}{$\mathrm{MCET}^{* *}$} & \multirow{2}{*}{$.0000^{*}$} \\
\hline No $(\%)$ & $300(100)$ & $350(100)$ & $200(100)$ & & \\
\hline \multicolumn{6}{|c|}{ Signing of front sheet: } \\
\hline $\begin{array}{c}\text { No }(\%) \\
\text { Not presen }\end{array}$ & $162(54)$ & $326(93)$ & $200(100$ & \multirow{3}{*}{ MCET** $^{* *}$} & \multirow{3}{*}{$.0000^{*}$} \\
\hline No $(\%)$ & $138(46)$ & $24(7)$ & $0(0)$ & & \\
\hline $\begin{array}{l}\text { Total } \\
\text { No }(\%)\end{array}$ & $300(100)$ & $350(100)$ & $200(100)$ & & \\
\hline \multicolumn{6}{|c|}{ Discharge time: } \\
\hline $\begin{array}{c}\text { No }(\%) \\
\text { Not presen }\end{array}$ & $0(0)$ & $185(53)$ & $82(41)$ & \multirow{3}{*}{$\mathrm{MCET}^{* *}$} & \multirow{3}{*}{$.0000^{*}$} \\
\hline $\begin{array}{l}\text { No }(\%) \\
\text { Total }\end{array}$ & $300(100)$ & $165(47)$ & $118(59)$ & & \\
\hline No $(\%)$ & $300(100)$ & $350(100)$ & $200(100)$ & & \\
\hline \multicolumn{6}{|c|}{$\begin{array}{l}\text { Discharge date: } \\
\text { Present }\end{array}$} \\
\hline $\begin{array}{c}\text { No }(\%) \\
\text { Not presen }\end{array}$ & $135(45)$ & $245(70)$ & $158(79)$ & & \\
\hline No $(\%)$ & $165(55)$ & $105(30)$ & $42(21)$ & 71.2 & $.0000^{*}$ \\
\hline $\begin{array}{l}\text { Total } \\
\text { No }(\%)\end{array}$ & $300(100)$ & $350(100)$ & $200(100)$ & & \\
\hline $\begin{array}{l}\text { Final diagnos } \\
\text { Present }\end{array}$ & & & & & \\
\hline $\begin{array}{c}\text { No }(\%) \\
\text { Not presen }\end{array}$ & $121(40.5)$ & $192(55)$ & $156(78)$ & & \\
\hline No $(\%)$ & $179(59.5)$ & $158(45)$ & $44(22)$ & 68.9 & $.0000 *$ \\
\hline Total & & & & & \\
\hline No (\%) & $300(100)$ & $350(100)$ & $22(100)$ & & \\
\hline
\end{tabular}

*Significant $(p$-value).

***Monte Carlo Exact test. 
Table (3): Comparison of completeness of documentation for items of discharge summary in the three studied hospitals.

\begin{tabular}{|c|c|c|c|c|}
\hline Variable & $\begin{array}{c}\text { Kafr } \\
\text { El-Sheikh } \\
\text { General Hospital }\end{array}$ & $\begin{array}{c}\text { El-Mahalla } \\
\text { El-Qubra } \\
\text { General Hospital }\end{array}$ & $\mathrm{X}^{2}$ & $p$ \\
\hline \multicolumn{5}{|c|}{ Patient status at discharge: } \\
\hline \multicolumn{5}{|c|}{ Present } \\
\hline No (\%) & $91(30.5)$ & $162(81)$ & & \\
\hline \multicolumn{5}{|c|}{ Not present } \\
\hline No (\%) & $209(69.5)$ & $38(19)$ & 123.2 & $.0000^{*}$ \\
\hline \multicolumn{5}{|l|}{ Total } \\
\hline No (\%) & $300(100$ & $200(100)$ & & \\
\hline \multicolumn{5}{|c|}{ Final diagnosis: } \\
\hline \multicolumn{5}{|c|}{ Present } \\
\hline No (\%) & $66(22)$ & $150(75)$ & & \\
\hline \multicolumn{5}{|c|}{ Not present } \\
\hline No (\%) & $234(78)$ & $50(25)$ & 137.4 & $.0000^{*}$ \\
\hline \multicolumn{5}{|l|}{ Total } \\
\hline No (\%) & $300(100)$ & $200(100)$ & & \\
\hline \multicolumn{5}{|c|}{ Operative procedures and } \\
\hline \multicolumn{5}{|c|}{ intervention: } \\
\hline \multicolumn{5}{|l|}{ Present } \\
\hline No (\%) & $101(33.5)$ & $4(2)$ & & \\
\hline \multicolumn{5}{|c|}{ Not present } \\
\hline No (\%) & $199(66.5)$ & $196(98)$ & 72.5 & $.0000^{*}$ \\
\hline \multicolumn{5}{|l|}{ Total } \\
\hline No (\%) & $300(100)$ & $200(100)$ & & \\
\hline \multicolumn{5}{|c|}{ Discharge medications: } \\
\hline \multicolumn{5}{|c|}{ Present } \\
\hline No (\%) & $66(22)$ & $111(55.5)$ & & \\
\hline \multicolumn{5}{|c|}{ Not present } \\
\hline No (\%) & $234(78)$ & $89(44.5)$ & 58.9 & $.0000^{*}$ \\
\hline \multicolumn{5}{|l|}{ Total } \\
\hline No (\%) & $300(100)$ & $200(100)$ & & \\
\hline \multicolumn{5}{|c|}{ Discharge instructions: } \\
\hline \multicolumn{5}{|c|}{ Present } \\
\hline No (\%) & $5(1.5)$ & $0(0)$ & & \\
\hline \multicolumn{5}{|c|}{ Not present } \\
\hline No $(\%)$ & $295(98.5)$ & $200(100)$ & $\mathrm{FE}^{* *}$ & 0.163 \\
\hline \multicolumn{5}{|l|}{ Total } \\
\hline No (\%) & $300(100)$ & $200(100)$ & & \\
\hline \multicolumn{5}{|c|}{ Follow-up appointments: } \\
\hline \multicolumn{5}{|c|}{ Present } \\
\hline No (\%) & $76(25.5)$ & $33(16.5)$ & & \\
\hline \multicolumn{5}{|c|}{ Not present } \\
\hline No (\%) & $224(74.5)$ & $167(83.5)$ & 5.5 & .0191 \\
\hline \multicolumn{5}{|l|}{ Total } \\
\hline No (\%) & $300(100)$ & $200(100)$ & & \\
\hline
\end{tabular}

*Significant ( $p$-value). $\quad * *$ Fisher's Exact test.

N.B: El-Obor Health Insurance Hospital is not included in the comparison of completeness of documentation items of discharge summary due to absence of discharge summary sheet. 
Table (4): Comparison of completeness of documentation for items of medical history sheet and physical examination form in the three studied hospitals.

\begin{tabular}{|c|c|c|c|c|c|}
\hline Variable & $\begin{array}{c}\text { Kafr } \\
\text { El-Sheikh } \\
\text { General Hospital }\end{array}$ & $\begin{array}{c}\text { El-Obor } \\
\text { Health Insurance } \\
\text { Hospital }\end{array}$ & $\begin{array}{c}\text { El-Mahalla } \\
\text { El-Qubra } \\
\text { General Hospital }\end{array}$ & $\mathrm{X}^{2}$ & $p$ \\
\hline \multicolumn{6}{|c|}{ Patient chief complaint: } \\
\hline \multicolumn{6}{|c|}{ Present } \\
\hline No $(\%)$ & $278(92.5)$ & $317(90.5)$ & $121(60.5)$ & \multirow{4}{*}{111.5} & \multirow{4}{*}{$.0000^{*}$} \\
\hline $\begin{array}{l}\text { Not presen } \\
\text { No }(\%)\end{array}$ & $22(7.5)$ & $33(9.5)$ & $79(395)$ & & \\
\hline \multirow{3}{*}{\multicolumn{6}{|c|}{$\begin{array}{l}\text { Total } \\
\text { No }(\%)\end{array}$}} \\
\hline & & & & & \\
\hline & & & & & \\
\hline \multicolumn{6}{|l|}{ Present } \\
\hline No $(\%)$ & $168(56)$ & $241(69)$ & $0(0)$ & & \\
\hline \multicolumn{6}{|c|}{ Not present } \\
\hline No $(\%)$ & $132(44)$ & $109(31)$ & $200(100)$ & $\mathrm{MCET}^{* *}$ & $.0000 *$ \\
\hline \multicolumn{6}{|l|}{ Total } \\
\hline No $(\%)$ & $300(100)$ & $350(100)$ & $200(100)$ & & \\
\hline \multicolumn{6}{|l|}{ Past history: } \\
\hline \multicolumn{6}{|l|}{ Present } \\
\hline No $(\%)$ & $228(76)$ & $156(44.5)$ & $56(28)$ & & \\
\hline \multicolumn{6}{|c|}{ Not present } \\
\hline No $(\%)$ & $72(24)$ & $194(55.5)$ & $144(72)$ & 123.1 & $.0000^{*}$ \\
\hline \multicolumn{6}{|l|}{ Total } \\
\hline No $(\%)$ & $300(100)$ & $350(100)$ & $200(100)$ & & \\
\hline \multicolumn{6}{|c|}{ Family history: } \\
\hline \multicolumn{6}{|c|}{ Present } \\
\hline No $(\%)$ & $242(80.5)$ & $194(55.5)$ & $117(57.5)$ & & \\
\hline \multicolumn{6}{|c|}{ Not present } \\
\hline No $(\%)$ & $58(19.5)$ & $156(44.5)$ & $83(41.5)$ & 43.1 & $.0000^{*}$ \\
\hline \multicolumn{6}{|l|}{ Total } \\
\hline No $(\%)$ & $300(100)$ & $350(100)$ & $200(100)$ & & \\
\hline \multicolumn{6}{|c|}{ Personal habits: } \\
\hline \multicolumn{6}{|c|}{ Present } \\
\hline No $(\%)$ & $71(23.5)$ & $16(4.5)$ & $32(16)$ & & \\
\hline \multicolumn{6}{|c|}{ Not present } \\
\hline No $(\%)$ & $229(76.5)$ & $334(95.5)$ & $168(84)$ & 49.8 & $.0000 *$ \\
\hline \multicolumn{6}{|l|}{ Total } \\
\hline No $(\%)$ & $300(100)$ & $350(100)$ & $200(100)$ & & \\
\hline \multicolumn{6}{|c|}{$\begin{array}{l}\text { Complete physical assessment } \\
\text { of all systems of the body: }\end{array}$} \\
\hline \multicolumn{6}{|c|}{ Present } \\
\hline No $(\%)$ & $208(69.5)$ & $311(89)$ & $180(90)$ & & \\
\hline Not presen & & & & & \\
\hline No $(\%)$ & $92(30.5)$ & $39(11)$ & $20(10)$ & 52.9 & $.0000 *$ \\
\hline Total & & & & & \\
\hline No $(\%)$ & $300(100)$ & $350(100)$ & $200(100)$ & & \\
\hline $\begin{array}{l}\text { Previous hosp } \\
\text { admissions an }\end{array}$ & & & & & \\
\hline Present & & & & & \\
\hline No $(\%)$ & $84(28)$ & $17(5)$ & $8(4)$ & & \\
\hline Not presen & & & & & \\
\hline No $(\%)$ & $216(72)$ & $333(95)$ & $192(96)$ & 95.6 & $.0000^{*}$ \\
\hline Total & & & & & \\
\hline No $(\%)$ & $300(100)$ & $350(100)$ & $200(100)$ & & \\
\hline
\end{tabular}

*Significant ( $p$-value).

**Monte Carlo Exact test. 
Table (5): Comparison of completeness of documentation for items of nursing notes in the three studied hospitals.

\begin{tabular}{|c|c|c|c|c|c|}
\hline Variable & $\begin{array}{c}\text { Kafr } \\
\text { El-Sheikh } \\
\text { General Hospital }\end{array}$ & $\begin{array}{c}\text { El-Obor } \\
\text { Health Insurance } \\
\text { Hospital }\end{array}$ & $\begin{array}{c}\text { El-Mahalla } \\
\text { El-Qubra } \\
\text { General Hospital }\end{array}$ & $\mathrm{X}^{2}$ & $p$ \\
\hline $\begin{array}{l}\text { Nursing pro } \\
\text { are recordea } \\
\text { Present }\end{array}$ & & & & & \\
\hline No $(\%)$ & $239(79.5)$ & $289(82.5)$ & $176(88)$ & & \\
\hline \multicolumn{6}{|c|}{ Not present } \\
\hline No $(\%)$ & $61(20.5)$ & $61(17.5)$ & $24(12)$ & 5.9 & $0.053 *$ \\
\hline \multicolumn{6}{|l|}{ Total } \\
\hline No $(\%)$ & $300(100)$ & $350(100)$ & $200(100)$ & & \\
\hline \multicolumn{6}{|c|}{ Legible medication name: } \\
\hline No $(\%)$ & $277(92.5)$ & $339(97)$ & $198(99)$ & & \\
\hline \multicolumn{6}{|c|}{ Not present } \\
\hline No $(\%)$ & $23(7.5)$ & $11(3)$ & $2(1)$ & 14.9 & $.0000^{*}$ \\
\hline \multicolumn{6}{|l|}{ Total } \\
\hline No $(\%)$ & $300(100)$ & $350(100)$ & $200(100)$ & & \\
\hline \multicolumn{6}{|l|}{ Dose: } \\
\hline \multicolumn{6}{|l|}{ Present } \\
\hline No $(\%)$ & $186(62)$ & $224(64)$ & $170(85)$ & & \\
\hline \multicolumn{6}{|c|}{ Not present } \\
\hline No $(\%)$ & $114(38)$ & $126(36)$ & $30(15)$ & 34.2 & $.0000 *$ \\
\hline \multicolumn{6}{|l|}{ Total } \\
\hline No $(\%)$ & $300(100)$ & $350(100)$ & $200(100)$ & & \\
\hline \multicolumn{6}{|l|}{ Route: } \\
\hline \multicolumn{6}{|l|}{ Present } \\
\hline No $(\%)$ & $159(53)$ & $285(81.5)$ & $153(76.5)$ & & \\
\hline \multicolumn{6}{|c|}{ Not present } \\
\hline No $(\%)$ & $141(47)$ & $65(18.5)$ & $47(23.5)$ & 67.4 & $.0000^{*}$ \\
\hline \multicolumn{6}{|l|}{ Total } \\
\hline No $(\%)$ & $300(100)$ & $350(100)$ & $200(100)$ & & \\
\hline \multicolumn{6}{|l|}{ Frequency: } \\
\hline \multicolumn{6}{|l|}{ Present } \\
\hline No $(\%)$ & $180(60)$ & $182(52)$ & $97(48.5)$ & & \\
\hline \multicolumn{6}{|c|}{ Not present } \\
\hline No $(\%)$ & $120(40)$ & $168(48)$ & $103(51.5)$ & 7.4 & $0.025^{*}$ \\
\hline \multicolumn{6}{|l|}{ Total } \\
\hline No (\%) & $300(100)$ & $350(100)$ & $200(100)$ & & \\
\hline
\end{tabular}

* Significant $(p$-value).

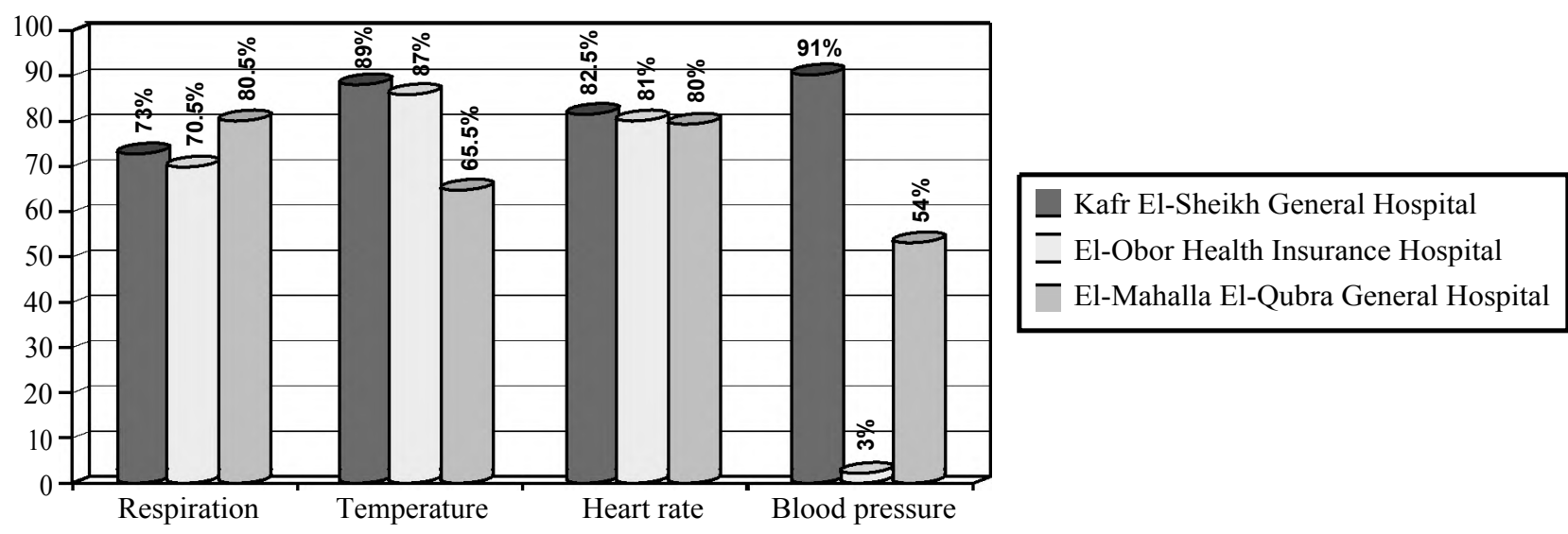

Fig. (1): Percentage of completeness of vital signs documentation sub items in the three studied hospitals. 


\section{Discussion}

In the current study, all basic forms were completely present in the three hospitals, except ElObor Health Insurance Hospital where general consent form and discharge summary were completely absent and routine diagnostic reports were present in more than the half of the checked records in the three studied hospitals. These findings are more better than the results of another study conducted in a training hospital in Iran, (2015) which showed that presence of discharge summary sheet and progress notes sheet were less than $50 \%$. Presence of medical history sheet was around $80 \%$ [6]. When compared with study done in Netherland, (2011), the presence of nursing notes sheet was unavailable in $1 \%$ of the patient records and the treatment order sheet in $21 \%$ of the reviewed patient records [7].

Almost all documentation items of patient identification part were present in more than $70 \%$ of checked records in El-Obor Health Insurance Hospital. This finding is consistent with the results of another study conducted in Iran, (2016) where it showed that data registration average in patient identification part of the teaching or University Hospitals was $53 \%$ and in other hospitals it was $52 \%$ [8]. In another study in Iran, (2017) that showed that mean documentation of demographic characteristics of the patient was 49\% [9]. In our study, documentation of provisional diagnosis in the front sheet was $92 \%$ in El-Obor Health Insurance Hospital and 96\% in El-Mahalla El-Kubra General Hospital. These findings are in accordance with the results of another study conducted in South India, (2013) showed that documentation of provisional diagnosis was from 70\%-90\% [10] Another study conducted in Nigeria, (2012). It revealed that provisional diagnosis was documented in $94.2 \%$ of records [11]

Documentation of final diagnosis in the front sheet was $40.5 \%$ in Kafr El-Sheikh General Hospital, 55\% in El-Obor Health Insurance Hospital and $78 \%$ in El-Mahalla El-Kubra General Hospital. Somi conducted a study to determine documentation of patient records at the internal medicine ward in Iran, (2004), showed that the final diagnoses were documented on $80.8 \%$ of the admission and discharge sheet [12]. Documentation of patient status at discharge was 30.5\% in Kafr El-Sheikh General Hospital while follow-up appointments was $25.5 \%$ and $16.5 \%$ in El-Mahalla El-Kubra General Hospital. In comparison with the results of another study conducted in Columbia, (2015), that revealed that presence of discharge status was
$30.71 \%$, outpatient follow-up appointments $51.5 \%$ and discharge medication list was recorded for almost all patients [13]

From a legal perspective, there was no form for general consents in El-Obor Health Insurance Hospital The percentage of general consent forms that were signed, was $63 \%$ in Kafr El-Sheikh General Hospital and 100\% in El-Mahalla ElKubra General Hospital. Pandit conducted a study in Nepal, (2010), revealed that the total number of medical records in the sample that had patient signature/finger print for treatment and release authorization forms was around $91 \%$ [14]

Documentation of complete physical assessment was present in $69.5 \%$ of checked records in Kafr El-Sheikh General Hospital, 89\% in El Obor Health Insurance Hospital and 90\% in El-Mahalla ElKubra General Hospital. These findings are higher than the results of another study conducted in a family health centre in El-Shorouk city, Egypt, (2016) where documentation of general examination was only in $51.5 \%$ of records [15]. Another study in USA, (2000) revealed only (28\%) of physical examination findings were documented [16]. On the other hand, another study conducted in Armenia, (2005), revealed that the mean percentage of completeness of physical examination sheet was $90 \%$ [17].

Documentation of the dose of the prescribed drugs was present in the majority of reviewed records in the three studied hospitals and that of route was 35\% in Kafr El-Sheikh General Hospital, $66.5 \%$ in El Obor Health Insurance Hospital and 59\% in El-Mahalla El-Kubra General Hospital. Documentation of the frequency of the prescribed drugs was present in almost of reviewed records in the three studied hospitals. Our study results are in accordance with the results of another study in Bahrain, (2008) where dosage of the prescribed drug, frequency and length of treatment were specified in $60.6 \%, 80.1 \%$ and $81.5 \%$ respectively [18] In the current study, illegible medication name was present in around $4.5 \%$ of reviewed records in the three studied hospitals. In comparison to the results of another study in Palestine, (2013), that revealed that the physicians' handwriting was poorly readable or illegible in one-third of the prescriptions. Drug dose and frequency were stated in $61.1 \%$ and $73.8 \%$ of prescriptions respectively [19].

Phalke conducted a study in India, (2011), showed that frequency, dose and route of administration of the drug were found on $73.7 \%, 64.9 \%$ and $75.2 \%$ prescriptions respectively [20]. Another 
study in Saudi Arabia, (2005), showed that in 6.5\% of prescriptions, the drug names were unreadable and the dose of prescribed drugs was mentioned in $80.6 \%$ of prescriptions [21]. Documentation of clinical progress notes is considered very poor in the three studied hospitals. Recording of clinical progress notes at least once a day was $31 \%$ in $\mathrm{Kafr}$ El-Sheikh General Hospital, 18.5\% in El Obor Health Insurance Hospital and 9\% in El-Mahalla El-Kubra General Hospital. On the other hand, in the study of assessment of medical records documentation in Iran, (2016) that showed that the average of data registration in the sheets of disease progress in teaching hospitals was $75 \%$ and $86 \%$ in non-teaching hospitals [8].

Recording of nursing progress notes after each shift was $79.5 \%$ in Kafr El-Sheikh General Hospital, $82.5 \%$ in El-Obor Health Insurance Hospital and $88 \%$ in El-Mahalla El-Kubra General Hospital. Another study conducted for evaluation of documentation at a hospital in Jamaica, (2013), it showed that $98 \%$ of the nurses provided a summary of the patients' condition at the end of each shift [22]. As regard to completeness of vital signs documentation items, blood pressure was documented only in 3\% of graphic charts in El-Obor Health Insurance Hospital and heart rate was documented in the majority of checked records $(80 \%)$ in the three studied hospitals. Documentation of patient's temperature was $65.5 \%$ in Kafr El-Sheikh General Hospital and $88 \%$ in the two remaining studied hospitals. Documentation of respiration was present in the majority of checked records $(75 \%)$ in the three studied hospitals. These findings are lower than the results of McGain's study in Australia, (2005) where respiratory rate was documented in $84.6 \%$, temperature was documented in $92.9 \%$, heart rate was documented in $95.8 \%$ and systolic blood pressure was documented in $96.3 \%$ of checked records [23]

As regard medication administration by the nurse, legible medication name administered was present in most of checked records $(95 \%)$ in the three studied hospitals. The documentation of the dose, route and frequency was present in majority of checked records $(70 \%),(75 \%)$ and $(53 \%)$ respectively of the three studied hospitals. These findings are higher than the results of Aitken's study in Australia, (2006) as the name of medication administered was only in $34 \%$ of medication administered charts and in $30 \%$ of charts, graduate nurses provided details about medications in a specific quantifiable way where the medication name, dose and frequency of administration were recorded [24]

\section{Conclusion:}

General consent form and discharge summary sheet were completely absent from the medical records of El-Obor Health Insurance Hospital. Almost all documentation assessment items of patient identification part were present in more than two third of checked records in El-Obor Health Insurance Hospital. The documentation assessment items of front sheet and discharge summary sheet were not present in more than the half of reviewed records at Kafr El-Sheikh General Hospital. Recording of clinical progress notes daily was not present in more than two third of reviewed records in the three hospitals.

\section{Recommendation:}

Targeted training programs for the medical staff at all levels regarding the importance of proper documentation. Conducting periodic audits of patient records to monitor content of medical care documented in patient records. Updating the documentation requirements of the current medical records forms in the three studied hospitals. A short course about medical records be included in the educational program of the students of medicine, this will help them to find out the important role of a complete medical record and their responsibility in relation to completeness of the record. For further research, comparative study of the current situation of existing medical records systems in Egyptian hospitals in different health care sectors is recommended.

\section{References}

1- Medical Records Manual: A Guide for Developing Countries. World Health Organization 2002. Revised and updated 2006.

2- ALI M. and HORIKOSHI Y.: Situation analysis of health management information system in Pakistan. Pakistan J. Med. Res., 41 (2): 64-69, 2002.

3- NASIRU S.I., HARUNA A.I., IDRIS M.A., SULEIMAN H. and BASHIR M.S.: Assessment of patient healthcare record management practices in tertiary health institutions of Jigawa state, Nigeria. International Journal of Advanced Technology and Science. 4 (5) May, 2016.

4- PANDIT U.: Study in the Quality of Clinical Documentation Practice in Chitwan Medical College Teaching Hospital, Nepal. Journal of Nepalgunj Medical College, 12 (2) December, 11-16, 2014.

5- ALI M. and KUROIWA C.: Accurate Record Keeping in Referral Hospitals in Pakistan's North West Frontier Province and Punjab: A Crucial Step Needed To Improve Maternal Health. J. Pak. Med. Assoc., 57: 443-46, 2007.

6- TORKI, SEDIGHE, NAHID TAVAKOLI and EL-AHE KHORASANI.: "Improving the Medical Record Documentation by Quantitative Analysis in a Training Hospital". Journal of Earth, Environment and Health Sciences, 1 (1): 22, 2015. 
7- ZEGERS M., de BRUIJNE M.C., SPREEUWENBERG P., et al.: Quality of patient record keeping: An indicator of the quality of care? BMJ. Quality \& Safety, 20: 314 $318,2011$.

8- SARAVI, BENYAMIN MOHSENI, et al.: "Documentation of medical records in hospitals of Mazandaran university of medical sciences in 2014: A quantitative study." Acta Informatica Medica, 24 (3): 202, 2016.

9- TARA, MAHMOOD and KOBRA ETMINANI: "Quantitative Evaluation Of Medical Record Documentation In Imam Reza Hospital, Mashhad, Iran." Medical Technologies Journal, 1 (4): 98-99, 2017.

10- SINHA, RAJESH KUMAR and NANDANA SHENOY.: "Assessment of oncology medical records as per NABH Standards." Journal of Health Management, 15 (3): 329343, 2013.

11- ADELEKE, IBRAHIM TAIWO, et al.: "Data quality assessment in healthcare: A 365-day chart review of inpatients' health records at a Nigerian tertiary hospital". Journal of the American Medical Informatics Association, 19.6: 1039-1042, 2012.

12- SOMI M.H., et al.: "Are the medical records of high quality in teaching hospitals?. " Journal of Medical Education, 5, 2, 2004.

13- AL-DAMLUJI, MOHAMMED SALIM, et al.: "Hospital variation in quality of discharge summaries for patients hospitalized with heart failure exacerbation". Circulation: Cardiovascular Quality and Outcomes Circoutcomes, 114, 2015.

14- PANDIT and UPENDRA.: "Study in the Quality of Clinical Documentation Practice in Chitwan Medical College Teaching Hospital, Nepal". Journal of Nepalgunj Medical College, 12 (2): 11-16, 2010.

15- ANWAR, WAGIDA A., et al.: "Measurement of Completeness of Medical Records in Family Health Centre in
El-Shorouk City". Egyptian Journal of Community Medicine, 34, 3, 2016.

16- SOLOMON, DANIEL H., et al.: "Can history and physical examination be used as markers of quality? An analysis of the initial visit note in musculoskeletal care". Medical care, 383-391, 2000.

17- ABRAHAMYAN, LUSINE, et al.: "One Year Event-free Survival Following Coronary Stent Revascularization In Nork Marash Medical Center, Yerevan, Armenia". Center for Health Services Research and Development, 2005.

18- AL-KHAJA K.A.J., et al.: "Prescription writing skills of residents in a family practice residency programme in Bahrain." Postgraduate Medical Journal, 84 (990): 198204, 2008

19- TAYEM Y.I., et al.: "Compliance with the guidelines of prescription writing in a central hospital in the West Bank". Eastern Mediterranean Health Journal, 19, 9, 2013.

20- PHALKE, VAISHALI D., et al.: "Prescription writing practices in a rural tertiary care hospital in Western Maharashtra, India." The Australasian Medical Journal, 4 (1): 4, 2011.

21- IRSHAID Y.M., et al.: "Compliance with good practice in prescription writing at outpatient clinics in Saudi Arabia", 2005.

22- BLAKE-MOWATT C., J.L.M. LINDO and J. BENNETT.: "Evaluation of registered nurses' knowledge and practice of documentation at a Jamaican hospital". International nursing review, 60 (3): 328-334, 2013.

23- McGAIN, FORBES, et al.: "Documentation of clinical review and vital signs after major surgery". Med. J. Aust., 189 (7): 380-3, 2008.

24- AITKEN, ROBYN, ELIZABETH MANIAS and TRCISHA DUNNING.: "Documentation of medication management by graduate nurses in patient progress notes: A way forward for patient safety". Collegian, 13 (4): 5-11, 2006. 


\section{تقييه وثائق السجلات الطبية فى مرافق الرعاية الصحية الثانوية

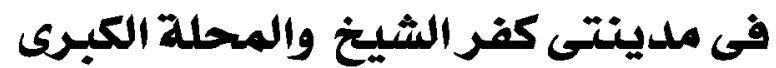

المقدمة: تلعب بيانات الرعاية الصحية الدقيقة والتى يسهل الوصول إليها دوراً هاماً فى تخطيط خدمات الرعاية الصحية وتطويرها وصيانتها. هدف البحث: تقييم جودة توثيق السجلات الطبية فى قسم السجلات الطبية فى ثلاث مرافق صحية، أثنان منهم فى مدينة كفر الشيخ الثيخ

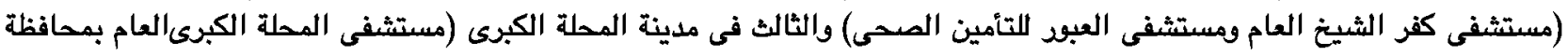
الغربية).

طريقة البحث: لتقييم جودة وثائق السجلات الطبية تم أختيار عينة عشوائية منهجية محسوبة من السجلات الطبية لكل مستشفى من سجلات عام r.

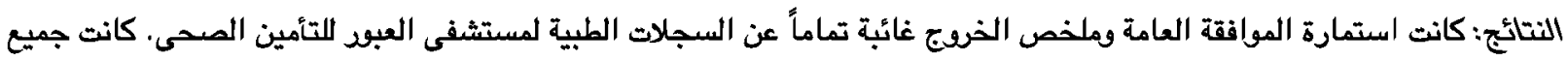

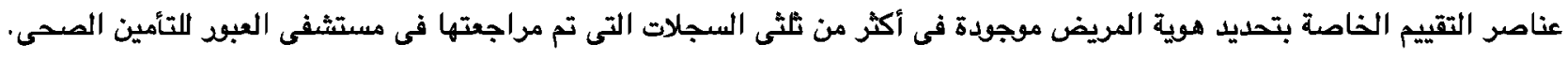

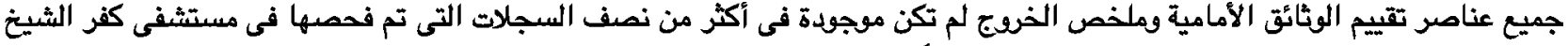

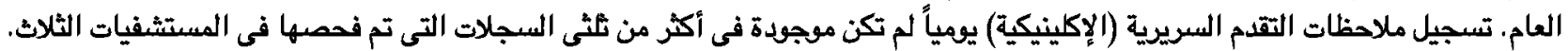

الخلاصة: كان هناك أختلافات كبيرة بين المستشفيات الثلاثة التى تمت الدراسة بها وفقاً لجودة التوثيق. 